\title{
Late-onset Candida keratitis after Descemet stripping automated endothelial keratoplasty: clinical and confocal microscopic report
}

\author{
Amanda Ortiz-Gomariz', Alejandro Higueras-Estebann", Ángel Ramón Gutiérrez-Ortega ${ }^{1}$, \\ José M. González-Méijome², Angeles Arance-Gil ${ }^{3}$ César Villa-Collar ${ }^{3}$ \\ ${ }^{1}$ Hospital General Reina Sofia, Murcia - Spain \\ ${ }^{2}$ Clinical \& Experimental Optometry Research Lab (CEORLab), Center of Physics, University of Minho, Braga - Portugal \\ ${ }^{3}$ Clínica Oftalmológica Novovision, Paseo de la Castellana, Madrid - Spain
}

\begin{abstract}
PURPOSE. To report clinical and confocal microscopy features of late-onset Candida albicans keratitis after Descemet stripping automated keratoplasty (DSAEK).

METHODS. We performed clinical and confocal scan on a patient who underwent DSAEK and phacoemulsification for Fuchs endothelial dystrophy and cataract.

RESULTS. A 76-year-old woman who underwent uneventful DSAEK and phacoemulsification presented with white to cream deposits 3 months after DSAEK. Confocal microscopy showed a hyperintense deposit at the lenticule and interface. Confocal scan also disclosed intense haze and inflammation at the interface and clusters of hyperreflective round structures resembling epithelial cells within the interface area. No signs of hyphae-like structures were seen. Late-onset symptoms misled us into a bacterial chronic endophthalmitis diagnosis, and the patient was started on topical and systemic antibiotics. Despite intense antibiotic therapy, the patient developed severe endophthalmitis, so we performed anterior vitrectomy and the donor lenticule was removed. Microbiology results from the removed lenticule showed infection by $C$ albicans. Antifungal therapy with systemic and topical voriconazole controlled the infection.

ConcLusIons. Candida interface keratitis is possible after DSAEK. The posterior location of infected tissue poses diagnostic and therapeutic challenges. In our case, the late onset of the symptoms and not performing corneoscleral rim cultures delayed correct diagnosis. This is the first reported case of post-DSAEK Candida keratitis with confocal microscopy images. A hyperintense granular deposit was seen at the lenticule and interface with confocal microscopy. We also observed intense haze, granular round structures resembling epithelial cells, and hyperreflective needle-shaped material at the interface. No hyphae-like structures were seen with confocal imaging.
\end{abstract}

KEY Words. Candida albicans, Confocal microscopy, DSAEK, Fungal keratitis

Accepted: December 8, 2010

\section{INTRODUCTION}

Candida keratitis is a rare complication of corneal transplantation and has been described after penetrating keratoplasty, anterior lamellar keratoplasty, and Descemet stripping keratoplasty $(\mathrm{DSAEK})(1,2)$.
Fungal keratitis accounts only for $1 \%-5 \%$ of cases of infectious keratitis in developed countries and approximately $10 \%-15 \%$ in developing countries (3). Fungal keratitis after keratoplasty may result from contaminated donor cornea or late inoculation in the presence of epithelial defects, sutures, and topical steroids. 
Although the frequency of fungal culture-positive donor corneoscleral rims has been reported to range from $0 \%$ to $12 \%(4,5)$, fungal infection incidence post penetrating keratoplasty is $0.16 \%$. In contrast, positive fungal donor rim culture is frequently associated with subsequent development of fungal keratitis of endophthalmitis. The odds of fungal infection with a fungal culture-positive donor rim are 247 times greater. In addition, clinical infection may occur several weeks or months after surgery despite an uneventful early postoperative course.

Another cause of fungal infection is preexisting epithelial defects and cornea invasion by conjunctiva and adnexa microflora, predominantly Candida species. Administration of topical corticosteroids enhances Candida replication through suppression of inflammatory host response.

Confocal microscopy has been used for real-time, noninvasive, in vivo observation of normal and pathologic cornea (6). Aspergillus keratitis in rabbit and human eyes has been reported (7). To our knowledge, this report is the first imaging late onset (3 months after surgery) Candida keratitis after DSAEK with confocal microscopy.

\section{Case report}

A 76-year-old woman with anterior narrow chamber, cataract, Fuchs endothelial dystrophy, and best-corrected visual acuity of 20/125 underwent an uneventful phacoemulsification and DSAEK surgery on the left eye on November 5, 2008.

The donor was a 44-year-old man who died of liver cirrhosis. The donor tissue was stored in Optisol GS for 7 days. The donor urine and ascitic fluid cultures were negative. All serologic tests including human immunodeficiency virus, hepatitis surface antigen, hepatitis $C$ virus, syphilis, and cytomegalovirus were negative.

During DSAEK, the donor corneal lenticule was prepared with a 350- $\mu \mathrm{m}$ head using the Moria anterior chamber maintainer. The posterior lamellar graft was punched from the endothelial side with a 7.75-mm Barron-Hessburg trephine and inserted with a 21-G vitrectomy forceps through a 4-mm clear corneal temporal incision without complication.

On the first postoperative day, the DSAEK graft was in good position, overlying edema and a $30 \%$ residual air bubble. The patient was started on prednisolone $1 \% 7$ times a day, ciprofloxacin $0.3 \% 5$ times a day, and $5 \%$ hypertonic sodium chloride eyedrops 5 times a day.

On November 19 (postoperative day 14), the DSAEK graft

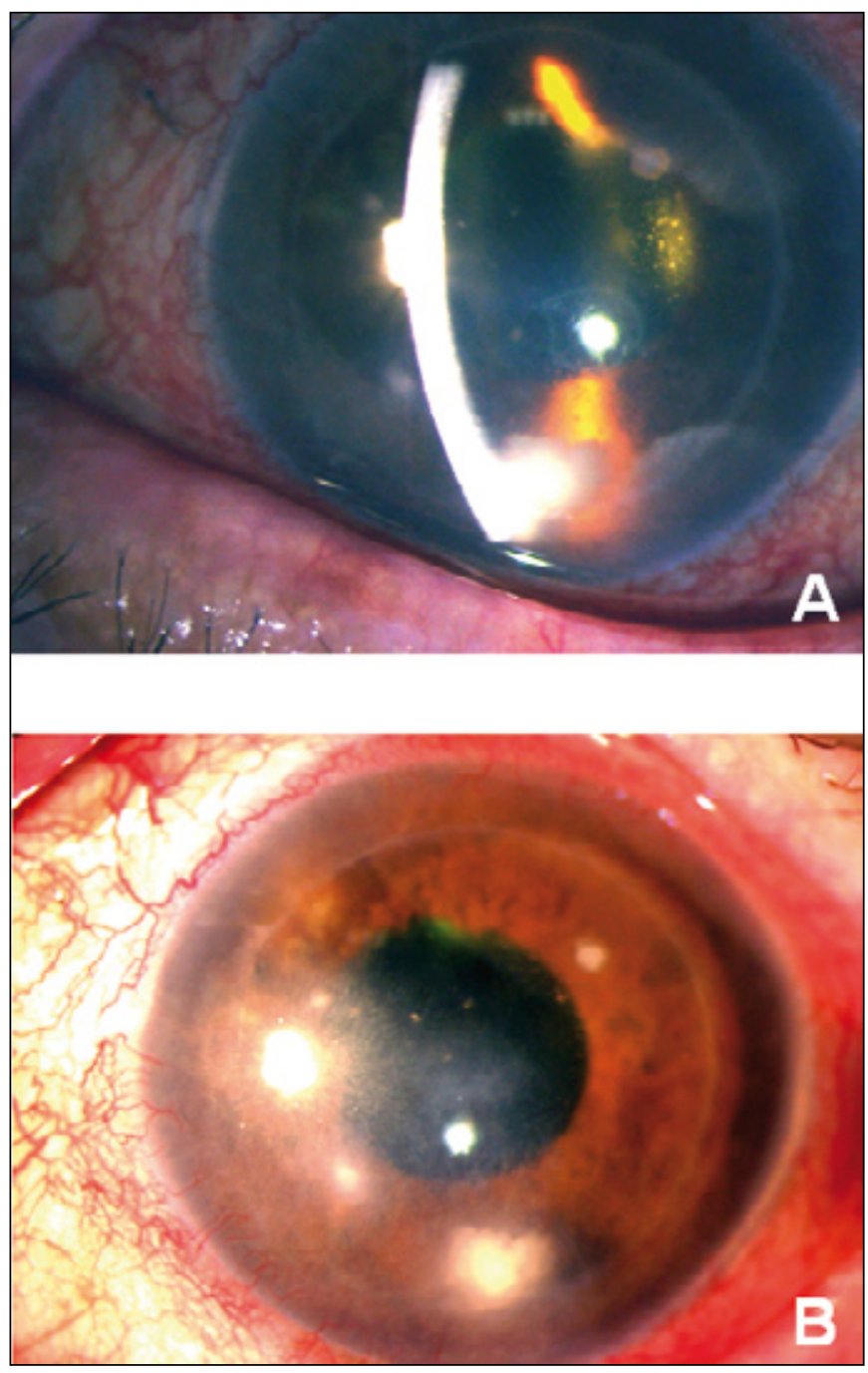

Fig. 1 - White-to-cream interface deposit (A); several satellite white deposits (B).

was clear with no evidence of corneal infiltrates, and the anterior chamber was quiet. Ciprofloxacin eyedrops were discontinued, and hypertonic sodium chloride and topical steroid prednisolone were gradually tapered to twice a day. During the next 2 postoperative months, the corneal graft remained clear and there was no corneal or anterior chamber inflammation. The best-corrected visual acuity was 20/70 in January 2009 in the left eye.

On February 4, 2009 (3 months after surgery), the patient returned with redness, tearing, and blurred vision in the left eye during the last 7 days. Scattered white-to-cream deposits appeared at the corneal interface region on slit-lamp microscopy (Fig. 1, A and B). The visual acuity dropped to 

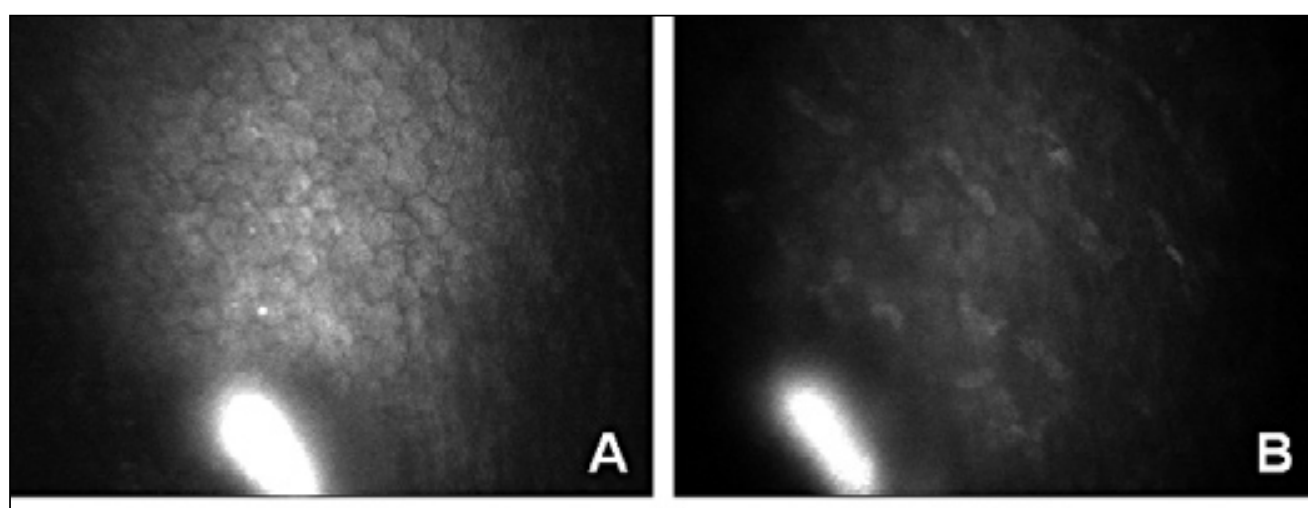

Fig. 2 - Hyperreflective granular deposit at donor endothelium (A); hyperreflective granular deposit at donor stroma (B); hyperreflective granular deposits at interface with intense haze and inflammation (C); hyperintense round structures and needle-shaped material at interface (D).

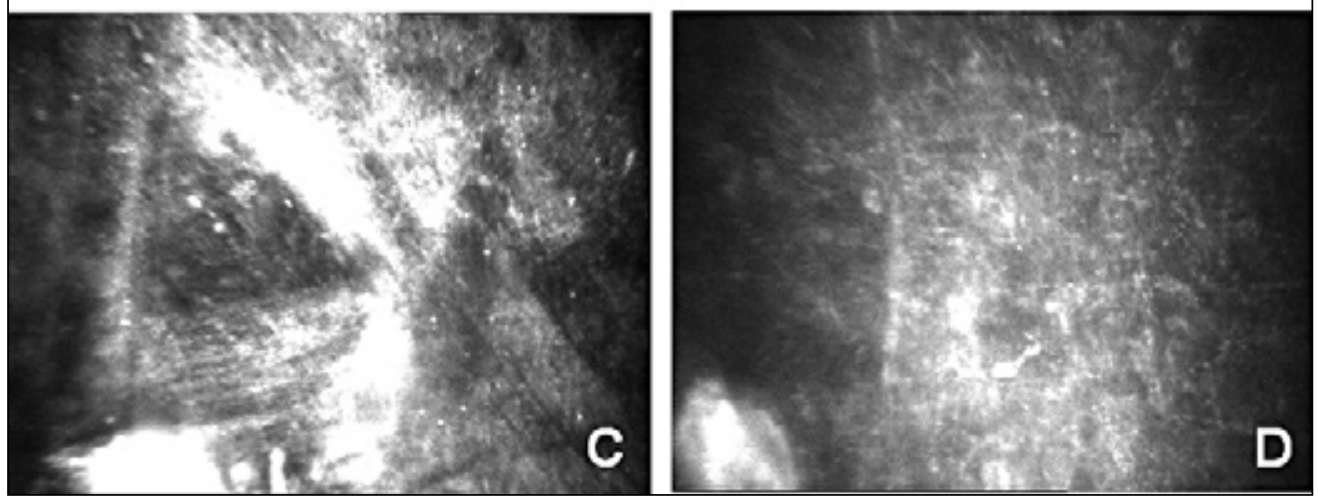

20/400 without correction, with no improvement on pinhole. Intraocular pressure (IOP) was $16 \mathrm{~mm} \mathrm{Hg}$. Chronic endophthalmitis was suspected and the patient was taken to the operating room on February 10, 2009. We performed extensive anterior chamber irrigation, aqueous humor culture was taken, and intravitreal vancomycin was injected. Postoperatively, the patient was continued on clarithromycin 500 mg twice a day and hourly vancomycin and amikacin eyedrops.

On confocal microscopy, we could observe a hyperreflective granular deposit between the donor lenticule and interface (Fig. 2, A-D). We could find evidence of inflammation at the interface area along with intense haze. We also observed needle-shape material at the interface and recipient stroma and hyperintense granular round structures resembling epithelial cells (Fig. 2, C and D). No hyphae-like structures were found.

Despite intense antibiotic therapy, the patient demonstrated an enlarging deep corneal infiltrate with overlying epithelial defect, hypopyon, increased corneal edema, and progressive vision loss to hand motion (Fig. 3A). B-scan ultrasonography showed evidence of posterior segment inflammation. Because of progression of the infiltrate, an- terior vitrectomy was performed and the donor lenticule was removed on February 27, 2009. During the surgery, aqueous and vitreous cultures were obtained and anterior chamber was irrigated with voriconazole, clindamycin, and vancomycin. Finally, intravitreous vancomycin and clindamycin were injected. We sent aqueous, vitreous, and donor lenticule to microbiology and cultures confirmed the presence of Candida albicans sensitive to voriconazole.

The patient was started on intravenous voriconazole 200 mg twice a day, oral corticosteroids, topical voriconazole every hour, vancomycin eyedrops every hour, clindamycin, dexamethasone eyedrops 4 times a day, timolol $0.5 \%$ eyedrops twice a day, and cycloplegic eyedrops 3 times day. Clinical appearance improved with intravenous and topical voriconazole and treatment was gradually tapered. Oral voriconazole was maintained during 6 weeks and topical voriconazole was maintained during 9 weeks.

On April 13, 2009, there were no signs of fungal infection, IOP was normal, but cornea was opaque. On May 27, 2009, we observed raised IOP and the patient was started on topical brimonidine twice a day, topical timolol twice a day, and topical dorzolamide twice a day. With this treatment, IOP reached normal values. On June 6, 2009, pen- 


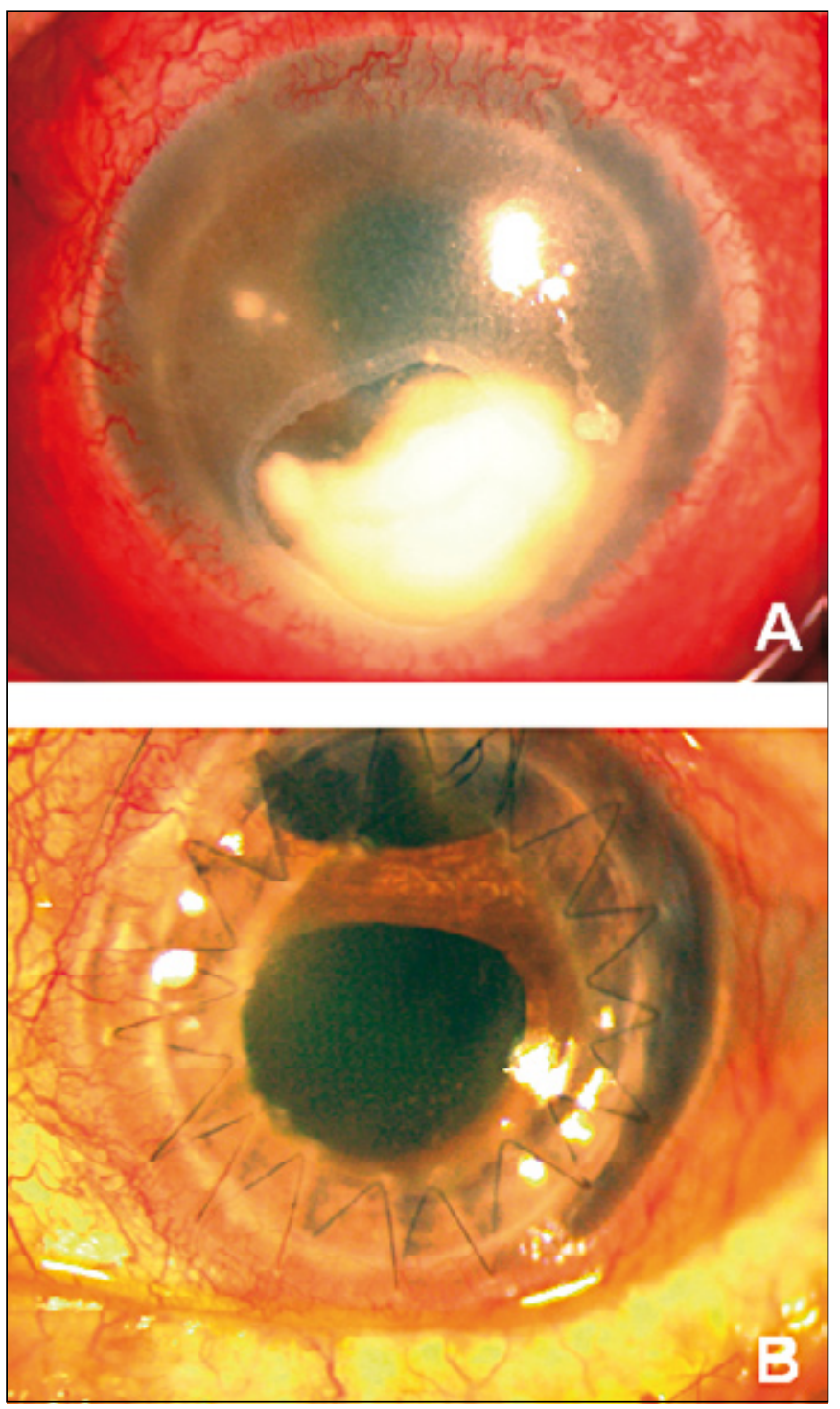

Fig. 3 - Enlarged and deep corneal infiltrate, overlying epithelial defect and hypopyon (A); clear cornea after penetrating keratoplasty and trabeculectomy (B).

etrating keratoplasty combined with trabeculectomy was performed.

On the most recent follow-up visit in March 2010 (9 months after penetrating keratoplasty), cornea remained clear, IOP was normal, and the best-corrected visual acuity on the left eye was 20/200. There has been no evidence of recurrent fungal keratitis (Fig. 3B).

\section{DISCUSSION}

Fungal keratitis is an uncommon complication after keratoplasty. Corneal transplantation is one of the risk factors for Candida keratitis, and concurrent administration of topical corticosteroids increases the risk of infection. Post-corneal graft Candida infection may occur because of donor corneal contamination or infection of preexisting epithelial defects by conjunctiva and adnexa microflora.

In this report, we present a patient with Candida endophthalmitis 3 months after DSAEK and phacoemulsification surgery. Donor rim culture is not currently performed in our patients; therefore, confirming transmission from donor to host was not possible. Candida contamination may have occurred during surgery as the donor endothelial lenticule is in contact with the conjunctiva before introducing it into the anterior chamber. There was no evidence of infectious keratitis or persistent epithelial defect in the donated fellow cornea after DSAEK.

Donor cornea lenticule infiltrates after DSAEK is a diagnostic and therapeutic challenge. The posterior location of infected tissue is a challenge in arriving at a correct diagnosis by cornea scraping. Obtaining diagnostic scraping and culture of infiltrated lenticule may be best achieved by removing the entire lenticule and culturing it on appropriate media. It is important to perform early removal of donor lenticule because this maneuver may help reduce the size of the infectious inoculum and may reduce the risk of intraocular infection. In addition, the effectiveness of topical antifungal agents may be reduced by posterior lamellar location of infiltrate and we may need to use systemic antifungal agents to achieve therapeutic aqueous concentrations. Furthermore, the presence of a posterior lamellar graft may sequester the infectious organism, leading to an atypical presentation of endophthalmitis with limited posterior segment inflammation.

The late onset of fungal infection in this case made diagnosis more difficult as the patient had been 3 months without any symptoms of fungal infection, which misled us in the differential diagnosis into bacterial chronic endophthalmitis. In another case reported by Chew et al (8), Candida parapsilosis was cultured from intraocular fluids in a case of early fungal endophthalmitis after DSAEK. The authors suggested that poor wound healing and wound dehiscence of the inferior venting incisions may have allowed intraocular entry of microorganisms. This mechanism is not likely to be present in the case reported here as venting 
incisions to reduce graft dislocation were not used in our surgical maneuvers.

In our case, confocal microscopy showed hyperreflective granular deposit in the donor lenticule and at the interface region. We also observed intense haze and inflammation at the interface along with high-contrast round structures resembling epithelial cells. We also found needle-shaped reflective material at the interface and host stroma, which is usually related to acute phase of corneal inflammation. We did not find evidence of hyphae-like formation. According to the present report, confocal microscopy is a powerful tool to identify and follow potential cases of infection after DSAEK. Chew et al (8) also pointed out this fact; however, those authors did not present confocal microscopy findings.

This patient also presented episodes of raised IOP potentially linked to narrow anterior chamber prior to DSAEK surgery so infection, chronic inflammation, and repeated surgeries produced angle closure glaucoma and angular synechiae. Performing penetrating keratoplasty and combined trabeculectomy finally controlled IOP.
In conclusion, fungal keratitis might occur several weeks or months after DSAEK and it must be included in the differential diagnosis for patients who present with interface infiltrates postoperatively. Confocal microscopy might be helpful in identifying uncommon features in the donor cornea and interface when suspecting the presence of infiltration or infection at different stages after DSAEK surgery.

The authors report no proprietary interest or financial support.

Address for correspondence:

José Manuel González-Méijome, PhD

Clinical \& Experimental Optometry Research Lab

Center of Physics (Optometry)

University of Minho

4710-057 Braga

Portugal

jgmeijome@fisica.uminho.pt

\section{REFERENCES}

1. Keyhani K, Seedor JA, Sah MK. The incidence of fungal keratitis and endophthalmitis following penetrating keratoplasty. Cornea 2005; 24: 288-91.

2. Koenig SB, Wirostko WJ, Fish RI, Covert DJ. Candida keratitis after Descemet stripping and automated endothelial keratoplasty. Cornea 2009; 28: 471-3.

3. Wilhelmus KR. Epidemiology of ocular infections. In: Baum J, Liesesang TJ, eds. Duane's Foundations of Clinical Ophthalmology, vol 2. Philadelphia: Lippincott Williams and Wilkins, 1998; 1-46.

4. Keyhany K, Seedor JA, Shah MK, Terraciano AJ, Ritterband DC. The incidence of fungal keratitis and en- dophthalmitis following penetrating keratoplasty. Cornea 2005; 24: 288-91.

5. Rehany $U$, Balut $\mathrm{G}$, Lefler $\mathrm{E}$, et al. The prevalence and risk factors for donor corneal button contamination and its association with ocular infection after transplantation. Cornea 2004; 23: 649-54.

6. Mastropasqua L, Nubile M. Confocal Microscopy of the Cornea. Thorofare, NJ: SLACK; 2002.

7. Avunduk AM, Beuerman RW, Varnell ED, et al. Confocal microscopy of Aspergillus fumigatus keratitis. $\mathrm{Br} \mathrm{J}$ Ophthalmol 2003; 87: 409-10.

8. Chew AC, Mehta JS, Li L, et al. Fungal endophthalmitis after Descemet stripping automated endothelial keratoplasty: a case report. Cornea 2010; 29: 346-9. 